\title{
The Comparison of Diagnostic Significance of Narrow Band Imaging in Contrast of White Light Endoscopy in the Assessment of Nasopharyngeal Cancer
}

\author{
Paudel Sharad1, Zhichun Huang1,2*, Xin Yang1, Wu Cao1,2, Ghimire Pratiksha1, Kharbuja Naresh1 \\ ${ }^{1}$ School of Medicine, South East University, Nanjing, China \\ ${ }^{2}$ Otorhinolaryngology and Head and Neck Surgery Department, Zhongda Hospital, Nanjing, China \\ Email: sharadpaudel123@gmail.com, ‘huang1963618@sohu.com,1614779643@qq.com,v-cw@sina.com, tuku255@gmail.com, \\ kharbuja_8@hotmail.com
}

How to cite this paper: Sharad, P., Huang, Z.C., Yang, X., Cao, W, Pratiksha, G. and Naresh, K. (2020) The Comparison of Diagnostic Significance of Narrow Band Imaging in Contrast of White Light Endoscopy in the Assessment of Nasopharyngeal Cancer. Journal of Cancer Therapy, 11, 160-171. https://doi.org/10.4236/jct.2020.113014

Received: March 3, 2020

Accepted: March 24, 2020

Published: March 27, 2020

Copyright $\odot 2020$ by author(s) and Scientific Research Publishing Inc. This work is licensed under the Creative Commons Attribution International License (CC BY 4.0).

http://creativecommons.org/licenses/by/4.0/

\section{Abstract}

Background: Narrow band imaging (NBI) is reported to improve the diagnostic importance of nasopharyngeal cancer. The purpose of this review was to evaluate the diagnostic significance of NBI in the literature and compare it to the conventional white light endoscopy. The use of narrow band imaging (NBI) and further technological achievements concerning the resolution and magnification of endoscopic images have in the past 15 years. With the use of NBI, superficial mucosal lesions, which may be missed by standard WLI endoscopy, can be identified easily by their neoangiogenic pattern. Objective: To assess diagnostic value of the narrow band imaging and white light endoscopy in nasopharyngeal carcinoma and compare diagnostic values (sensitivity, specificity, positive predictive value and negative predictive value) with white light endoscopy. Search Methods: From 2010 to 2020, data was searched from electronic databases such as PubMed, web of science. We used narrow band imaging as a key word accordance with diagnostic modalities such as sensitivity, specificity, PPV and NPV and data were collected. Results: We have found mainly 6 studies have discussed about diagnostic value of endoscopy in nasopharyngeal carcinoma in the total of 2746 suspected patients. Among them, 5 studies have compared diagnostic values such as sensitivity, specificity, positive predictive value and negative predictive value between NBI and WLE. Among 5 studies, 4 studies have found higher sensitivity in NBI, 2 studies found higher 1 equal to WLE specificity in NBI. 3 studies have compared PPV and NPV between NBI and WLE. Among them, all the studies found higher PPV and NPV in NBI than WLE. Conclusion: Recently 
developed narrow band imaging has a great significance in the diagnosis of nasopharyngeal carcinoma. Although NBI has also encountered some problem such as contact bleeding and darker image. So, further evaluation should be done.

\section{Keywords}

Narrow Band Imaging, White Light Endoscopy, Head and Neck Neoplasms, Nasopharyngeal Carcinoma, NBI Classification, IPCLs, Image Enhanced Endoscopy

\section{Introduction}

Nasopharyngeal carcinoma (NPC) is a tumor common in Southeast Asia. The region with the highest incidence is southern China, with 25/100,000 people in Guangzhou and Taiwan every year. The mean age at diagnosis was 45 - 55 years, with an annual incidence of 23.3 cases per 100,000 men and 8.9 cases per 100,000 women [1]. In China, the incidence varies from region to region and gradually decreases from South China to North China, with an annual incidence of 2 - 3 cases per 100,000 people. The geographic and ethnic distribution of this tumor is significant, with a particularly high prevalence in southern China, Southeast Asia, and North Africa [2].

In the present scenario, most of the medical institutes use white light endoscopy guided histopathological examination for the diagnosis of NPC [3]. Although larger tumor is easily seen by white light endoscopy, but superficial tumor is hard to be identified with WLE. Early and small tumors may not be distinguishable from adenoid tissue or normal nasopharyngeal mucosa. So, there is high chance of missing small or early nasopharyngeal tumors by WLE. Nasopharyngeal cancer and precancerous lesions are usually difficult to analysis and may pose a challenge for doctors trying to visualize them using standard white light endoscopes. To overcome this, new endoscopic method is developed color spectrum of tumor surface to visualize tumor microvascular patterns called image enhanced endoscopy [4] [5]. It uses optical and/or electronic methods, such as narrow-band imaging (NBI), auto fluorescence imaging (AFI), i-scans, flexible spectral imaging color enhancement (FICE), confocal laser endo microscopy (CLE) and I scan Optical Enhancement (OE) endoscopy.

Narrow-band imaging (NBI) is newly developed image enhanced technique developed by Olympus. It follows NBI principle which uses two specific wavelengths of light that are strongly absorbed by hemoglobin [6] [7]. NBI technology filters selected light waves (that is, $415 \mathrm{~nm}$ blue light and $540 \mathrm{~nm}$ green light) by using hemoglobin bandwidth adsorption. In this way, narrow band waves not only penetrate the epithelium to highlight the capillary network associated with neoangiogenesis, but also reach deeper tissues, thereby enhancing submucosal epithelial blood vessels, highlighting the capillary network, and penetrate deeper 
tissues to enhance visibility of the subepithelial vessels [8]. Narrow-band imaging has proven excellent diagnostic accuracy in distinguishing between various cancerous and pre-cancerous lesions. Since its inception, NBI has been used primarily for the detection of gastrointestinal diseases and malignancies [9]. In recent years, there have been some reports about the diagnosis of malignant tumors of the head and neck including nasopharyngeal carcinoma. NBI significantly improves the contrast of surface capillary patterns and makes it easier to detect small or early malignant lesions, thus improving the sensitivity and specificity of diagnosis. It is now widely used for the early detection of nasopharyngeal cancer, and it has higher sensitivity and specificity than traditional endoscopic white light imaging. Some recent reports describe its use in the early identification of nasopharyngeal cancer and its diagnostic value on nasopharyngeal cancer.

\section{Endoscopic Evaluation of Nasopharyngeal Carcinoma}

\subsection{Conventional White Light Endoscopy (WLE)}

White light endoscopy is the preferred initial study for detecting nasopharyngeal carcinoma (NPC). Endoscopic biopsy of the primary tumor site can confirm a clear diagnosis of NPC. White light endoscopes are often used to screen for nasopharyngeal cancer. There are some studies that show diagnostic significance of WLE for the diagnosis of nasopharyngeal carcinoma.

Wang Wen-Hung et al. [10] studied 106 patients with suspected nasopharyngeal carcinoma in 2011. All patients underwent routine nasopharyngeal white light endoscopy, and they identified 32 suspicious lesions in WLE by endoscopy. According to pathological data and clinical follow-up results, the diagnosis of recurrent nasopharyngeal carcinoma by conventional WL endoscope is as follows: sensitivity $37.5 \%$; specificity $92.9 \%$ negative predictive value $94.8 \%$. This study showed that the sensitivity and specificity of WL endoscopy in recurrent nasopharyngeal tumors were $37.5 \%$ and $92.9 \%$, respectively. Therefore, the main limitation of using standard WL endoscopes to monitor recurrent NPC is its low sensitivity of 37\%. Similarly, Yang Haidi et al. [11] in 2012 found 62 cases of pathologically confirmed nasopharyngeal carcinoma. The sensitivity, specificity, positive predictive value and negative predictive value of NPC were $90.3 \%$, $75.4 \%, 11.3 \%$ and $99.6 \%$, respectively. In this study, when the primary tumor was located under the nasopharyngeal mucosa or in the crypt of the pharynx (also known as Rosen Muller's fossa), it may not be seen on endoscopy. Patients with such tumors receive random endoscopic biopsies to sample the nasopharynx, but despite the sampling, some early tumors may be missed. Ching Yinho et al. [12] in 2013 studied 211 cases from 154 NPC families, of which the false positive rate of primary NPC diagnosed by conventional WLE was $8.9 \%-38 \%$ and the false negative rate was $0.4-5.35 .3 \%$, sensitivity was $62 \%-64.7 \%$, specificity was $91.1 \%-99.6 \%$, positive predictive value was $84.6 \%-98 \%$, and negative predictive value was $77.4 \%-88.9 \%$. In their results, primary NPC was diagnosed in a 
family of unaffected NPC patients by conventional WLE, with a false-positive rate of $22.2 \%$, a false-negative rate of $0 \%$, a sensitivity of $77.8 \%$, and a specificity of $100 \%$. The main limitation of WLE in this study was low sensitivity.

Although WLE is very useful for the early diagnosis of nasopharyngeal carcinoma, there are some limitations found during the studies. Wen-Hung Wang et al. in 2011 and ching yin ho in 2013 found the limitation of WLE is low sensitivity. Yang Haidi et al. in 2012 believed that primary tumour lies beneath the nasopharyngeal mucosa or within the pharyngeal recess; it may be miss at WL endoscopic inspection. Low sensitivity and missing early nasopharyngeal cancer are major disadvantage of WLE. So, further evaluation is needed for the diagnosis of nasopharyngeal carcinoma.

\subsection{Narrow Band Imaging (NBI)}

NBI systems available in South Korea (Evis Lucera Spectrum system, CV-260SL; Olympus Medical Systems Co., Ltd. in Tokyo, Japan) have filters that transmit only $415 \pm 15 \mathrm{~nm}$ and $540 \pm 15 \mathrm{~nm}$ wavelengths. Narrowband imaging (NBI) is a new optical technology that enhances the diagnostic sensitivity of endoscopes to tissue features by using narrow-bandwidth filters in continuous red, green, and blue lighting systems. The diagnosis of nasopharyngeal carcinoma is based on NBI classification of nasopharyngeal mucosal micro vessel patterns which is based on IPCLs [13] (Figure 1).

\section{Principle of Narrow Band Image}

NBI is a non-invasive technique for visualizing the structure of mucosal blood vessels. It relies on the principle of light penetration depth and hemoglobin absorption peak. The NBI system includes a special image processor and a lighting unit with a special filter, which can reduce the frequency range of white light to $400-430 \mathrm{~nm}$ (centered at $415 \mathrm{~nm}$, i.e. blue) and $525-555 \mathrm{~nm}$ (centered at 540 $\mathrm{nm}$, i.e. green)) band. Light at $415 \mathrm{~nm}$ (blue) has less penetrating power and less scattering, thus improving image resolution. $540 \mathrm{~nm}$ (green) light penetrates deeper, highlighting submucosal vascular plexus [14].

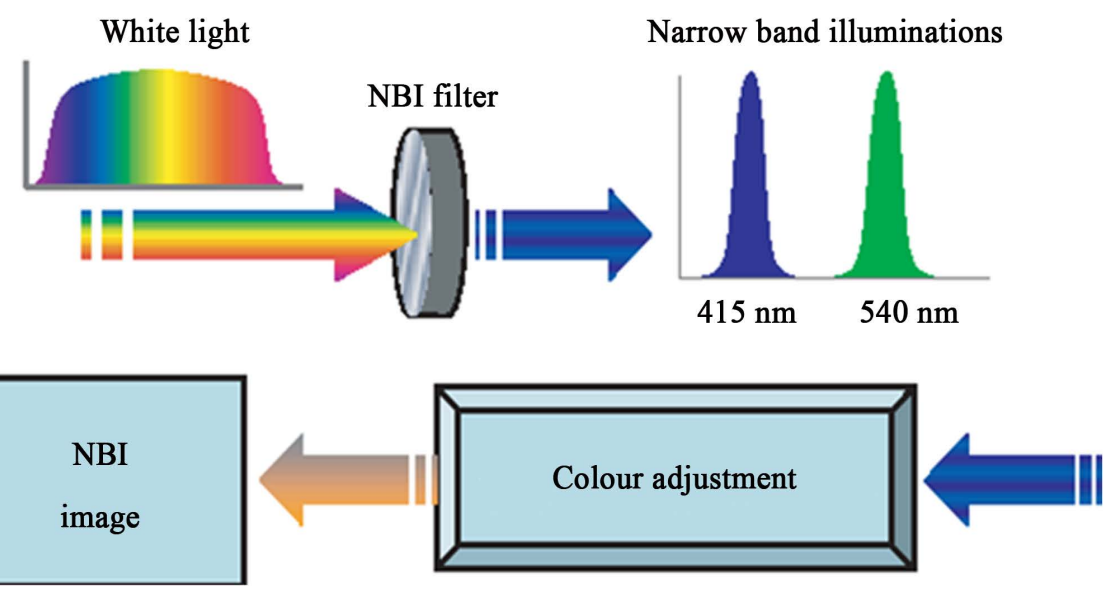

Figure 1. Narrow band imaging principle (picture courtesy: Alberto Larghi et al. [13]. 


\section{NBI Classification of Nasopharyngeal Mucosal Micro Vessel Patterns}

NBI classification under NBI is the key for diagnosis of NPC. Many authors have proposed the NBI classification of nasopharyngeal mucosal micro vessel patterns for NPC. The Yi-Hui Wen [15] in 2012 they classified the appearance of nasopharyngeal microvessels examined with NBI into 4 types (see Figure 2). Type I is short, sparse, and sparse, located between lymphoid follicles. Type II is moderate in length and diameter, regular reticulate; Type III has vascular bifurcation, dilation, elongation and mild irregularity; Type IV is deformed in a shape, and the diameter and blood vessel direction are very irregular. According to Yi hui wen, type I and type II microvessel patterns were considered as normal nasopharynx where microvessels with submucosal vessels observed as green and arborescent branches seen as brown in NBI. When lesion is developed to malignant, intra epithelial papillary and capillary loops (IPCLs) becomes more dilated, elongated and mildly irregular in type III and well demarcated brownish area with twisted into a spiral worm-like appearance in type IV.

Similarly, Wang Hung et al. [10] in 2012 proposed new NBI classifications for nasopharyngeal carcinoma. In this study, they made five distinct findings under NBI, Type I: Brown spots, Type II: Irregular microvascular pattern (IMVP), Type III: Shallow peak, Type IV: Side differences, type V: IMVP or side differences. Among $5 \mathrm{NBI}$ types, the last three (type III - V) were new concepts for the detection. But, in the clinical use, the wen NBI classification and wang NBI classification both were not easy to use because of its lacking of mucosal microvascular morphology of post radiated nasopharyngeal carcinoma. Later, Xiao Guang Ni et al. in [16] 2017 proposed new NBI classification of nasopharyngeal mucosal micro vessel patterns for NPC (Figure 3).
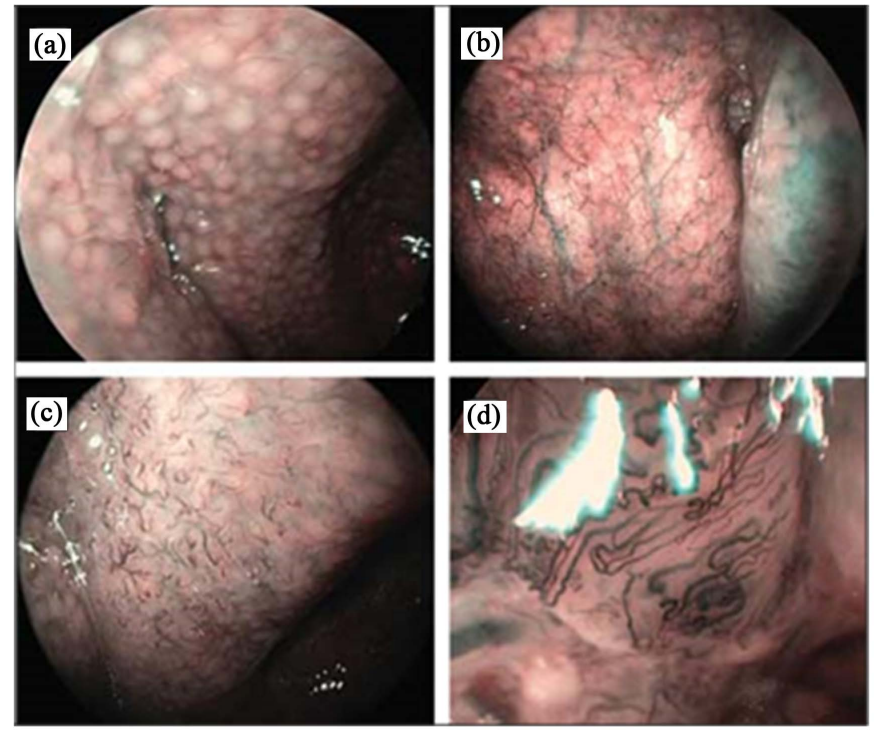

Figure 2. The Wen NBI classification ((a) type I: short, thin, and scanty; (b) type II: moderate length and diameter, regularly reticulate (c) type III: bifurcated, expanded and elongated, mild irregularity and (d) type IV: earthworm-like distortion with a very irregular diameter or vessel course) (Picture courtesy: Yi Hui Wen et al. [15]). 

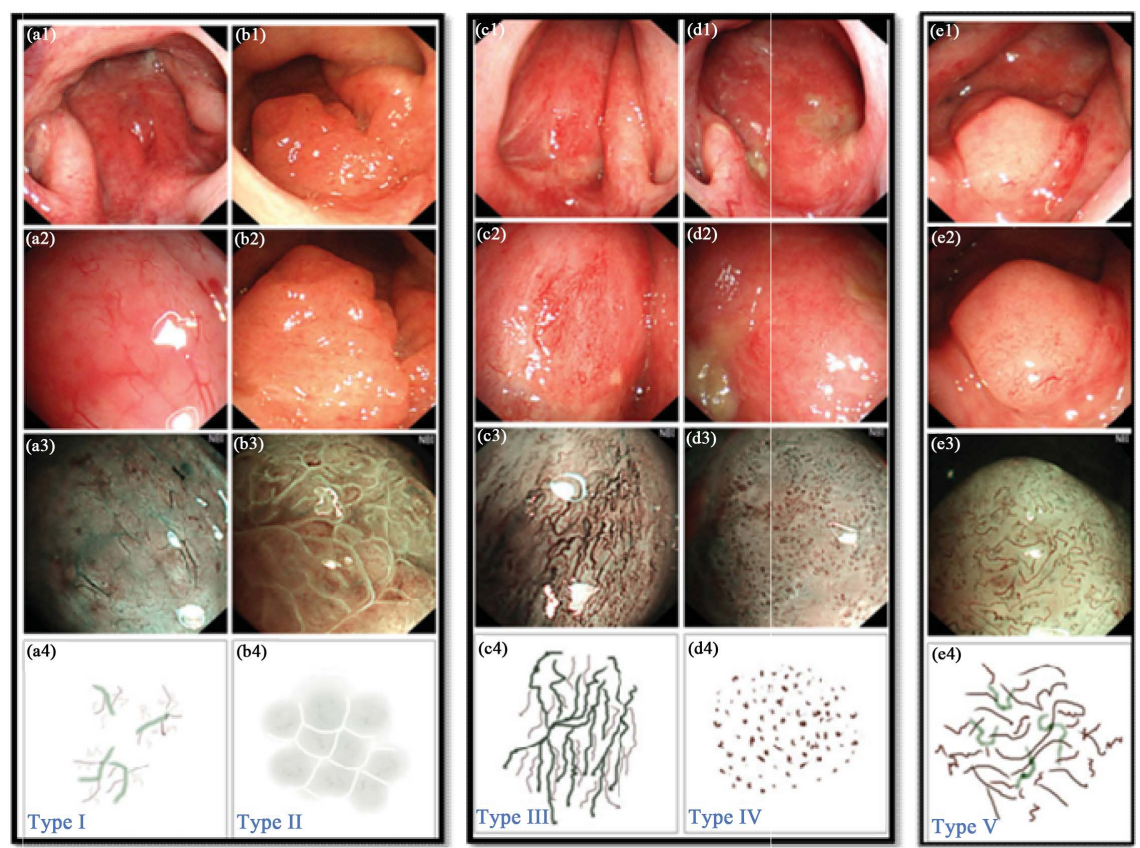

Figure 3. (a1) - (a4) type I microvessel pattern, normal nasopharyngeal mucosa; (b1) (b4) Type II microvessel pattern, lymphoid hyperplasia. (c1) - (c4) type III microvessel pattern, radiated inflammation; (d1) - (d4) type IV microvessel pattern, radiated inflammation, (e1) - (e4) Type V microvessel pattern, nasopharyngeal carcinoma (Picture courtesy: Xiao Guang Ni et al. [16]).

There are various studies of NBI on nasopharyngeal carcinoma according to these NBI classification of microvessel patterns. Ching Yin Ho et al. [17] in 2013 included 63 patients who were suspected nasopharyngeal carcinoma, the sensitivity, specificity, positive predictive value, and negative predictive value of NBI in nasopharynx (NP) were $78.0 \%, 95.5 \%, 97.0 \%$, and $70.0 \%$, respectively. J Madana et al. [18] in 2015 conducted a prospective cohort study of 53 redetection of early NPC Among 19 normal patients, common NBI features were discrete brown spots (100\%) and regular polygonal follicular pattern of nasopharyngeal mucosa (90\%). The features of 15 patients with NPC were loss of normal polygonal follicular architecture of nasopharynx (100\%), concentrated brown spots (93\%), and pale pink areas (80\%). Among 19 patients after NPC radiotherapy, the common features were patchy dark brown green spots (100\%), polygonal follicular loss of the nasopharyngeal mucosa (95\%), and discrete microvascular branches (95\%). Xiaoguang Ni et al. [16] in 2017 did the research on a total of 290 patients with a suspected nasopharyngeal tumour. On the result, he found that approximately $93.5 \%$ (29/31) of lymphoid hyperplasia appeared as the type II microvessel pattern under NBI endoscopy, whereas $96.2 \%$ (51/53) of nasopharyngeal radiation-induced inflammation exhibited the type III or IV microvessel pattern. The characteristics of NPC under NBI endoscopy mainly appeared as a type $\mathrm{V}$ microvessel pattern $(79.5 \%, 167 / 210)$, and the sensitivity, specificity, predictive value (PPV), and negative predictive value (NPV) of type $\mathrm{V}$ in the diagnosis of NPC were $79.5 \%, 91.3 \%, 96.0 \%$, and $62.9 \%$, respectively. 
In the conclusion, NBI is an innovative optical image enhancement technology that highlights the microsurface pattern and microvascular morphology of the mucosal surface. Although, NBI had high diagnostic values for the nasopharyngeal carcinoma, there were some limitations were found during the studies. Ching Yin Ho et al. in 2013 found some limitations such as secretion coating and bleeding during NBI endoscopy. J Madana et al. in 2015 found that a limitation encountered in our study was that the presence of mucus and blood significantly interfered with the observation of the NBI features. Xiao Guang Ni et al. found in 2017 that contact bleeding can interfere with the observation and cover up the microvascular morphology. They believe that the diagnosis determined by NBI endoscopy is a subjective judgment, which may lead to diagnostic bias and inaccuracy.

\section{Search Methods}

\subsection{Search Strategy}

From 2010 to 2020, data was searched from electronic databases such as PubMed, web of science to find studies in which NBI endoscopy was used to screen for nasopharyngeal carcinoma in compared to WLE. For this search we used the terms such as "narrow band imaging", "nasopharyngeal carcinoma" and "narrow band imaging vs White light". For the example in pubmed, we searched the key term "NBI" in nasopharyngeal carcinoma and "White light endoscopy" in nasopharyngeal carcinoma separately. Then, we combined these terms as an "and" and "or" search, yielding five articles which were specific for this study. We only included English articles with prospective trial of NBI vs WLE in suspected nasopharyngeal carcinoma. Reviewers were not blind to the author name, institution, or journals and references of this article were cited in various articles.

\subsection{Eligibility and Data Extraction}

We included all prospective studies using NBI to detect NPC compared to standard WL. Excluded criteria included reviews and data reported in abstract form only, non-diagnostic studies, studies that did not include histologically confirmed or extractable raw data, and retrospective studies. Data extraction was done by EXCEL. The inclusion and exclusion criteria are applied independently by two reviewers, and any differences of opinion are resolved through discussion between reviewers.

\section{Results}

By using NBI, superficial mucosal lesions that may be missed by standard WLI endoscopy can be easily identified through its neovascularization pattern. Early NPC may be in hidden locations, or often have a microscopic appearance of superficial inflammation that WLE is easily overlooked or misdiagnosed. For this lesion, NBI was found to be useful in the diagnosis of nasopharyngeal carcino- 
ma. Many authors believe that the sensitivity of traditional white light endoscopy is not sufficient to screen for superficial cancers or precancerous lesions of nasopharyngeal carcinoma (Table 1).

Yi Huiwen et al. [15] in 2012 included a total of 285 cases of suspected nasopharyngeal carcinoma, of which 66 cases were cancerous. Of the 66 cancer lesions diagnosed on pathological examination, 62 were found by NBI and only 47 by WLI. The detection sensitivity of NBI to NPC was significantly higher than that of WLI (93.9\% vs. $71.2 \%$; $\mathrm{P}=0.001)$. The specificity of NPC detected using NBI (206 [94.1\%] of 219] and WLI ([95.4\%] of 209 of 209; P = 0.52) was not significantly different. NBI (62 cases, 75 [82.7\%]) and WLI (47 cases, 57 [82.5\%]) had no significant difference in positive predictive value $(\mathrm{P}=0.98)$. The negative predictive value of NBI (210 of 210 [98.1\%]) was significantly higher than that of WLI (228 of 209 [91.7\%], P = 0.003). He concluded that due to enhanced visualization of epithelial and subepithelial microvascular patterns, narrowband imaging endoscopes have higher sensitivity and NPV, which can be used for early diagnosis of cancerous lesions, improve diagnostic accuracy. Similarly, Wen-Hung Wang et al. in 2012 [10] also performed a research on same 106 patients to compare the diagnostic values (sensitivity, specificity and diagnostic value) between NBI and WLE. The sensitivity, specificity, and diagnostic capability were $37.5 \%, 92.9 \%$ and $65.2 \%$ for WLE and for $87.5 \%, 74.5 \%$ and $81.0 \%$ for NBI respectively. Haidi Yang et al. in 2012 [11] performed a study in a total of 1854 patients suspected nasopharyngeal carcinoma (WL) and an NBI system to compare the diagnostic values (sensitivity, specificity, PPV and NPV). The sensitivity, specificity, positive predictive value and negative predictive value for detecting NPC significantly increased from $90.3 \%, 75.4 \%, 11.3 \%$ and $99.6 \%$ with WL up to $100 \%, 99.2 \%, 81.6 \%$ and $100 \%$ with NBI respectively.

Ching-Yin Ho et al. [12] in 2013 concluded a total of 211 patients suspecting NPC in their study. The sensitivity and specificity were $22.2 \%$ and $100 \%$ using conventional WLE, and $44.4 \%$ and $100 \%$ using NBI. Xiao Guang Ni et al. [16] in 2017 enrolled a total of 290 patients with a suspected nasopharyngeal tumour. The sensitivity, specificity, PPV, and NPV of type V in the diagnosis of NPC were $79.5 \%, 91.3 \%, 96.0 \%$, and $62.9 \%$, respectively. The diagnostic sensitivity, specificity, PPV, and NPV of WLI endoscopy for NPC were $85.2 \%, 51.3 \%$,

Table 1. The comparison of diagnostic values between NBI and WLE on suspecting NPC.

\begin{tabular}{|c|c|c|c|c|c|c|c|c|c|c|}
\hline \multirow{2}{*}{ Authors } & \multirow{2}{*}{$\begin{array}{c}\text { Year of } \\
\text { publication }\end{array}$} & \multirow{2}{*}{$\begin{array}{c}\text { No. of } \\
\text { samples }\end{array}$} & \multicolumn{4}{|c|}{ WLE (\%) } & \multicolumn{4}{|c|}{ NBI (\%) } \\
\hline & & & Sensitivity & Specificity & PPV & NPV & Sensitivity & Specificity & PPV & NPV \\
\hline Yi Hui Wen et al. [15] & 2012 & 285 & 71.2 & 95.4 & 82.5 & 91.7 & 93.9 & 94.1 & 82.7 & 98.1 \\
\hline Wen-Hung Wang et al. [10] & 2012 & 106 & 37.5 & 92.9 & N/A & N/A & 87.5 & 74.5 & N/A & N/A \\
\hline Hai Di Yang et al. [11] & 2012 & 1854 & 90.3 & 75.4 & 11.3 & 99.6 & 100 & 99.2 & 81.6 & $100 \%$ \\
\hline Ching Yin Ho et al. [12] & 2013 & 211 & 22.2 & 100 & N/A & N/A & 44.4 & 100 & N/A & N/A \\
\hline Xiao Guang Ni et al. ${ }^{*}[16]$ & 2017 & 290 & 85.2 & $51.3 \%$ & 82.1 & 59.6 & 79.5 & 91.3 & 96.0 & 62.9 \\
\hline
\end{tabular}

*Above data is comparison by Xiao Guang Ni et al. with type $\mathrm{V}$ microvessels (malignant). 
$82.1 \%$, and 59.6\%, respectively. The sensitivity, specificity, PPV, and NPV of type II in the diagnosis of nasopharyngeal lymphoid hyperplasia were $93.5 \%$, $84.9 \%, 42.6 \%$, and $99.1 \%$, respectively. The sensitivity, specificity, PPV, and NPV of WLI endoscopy in the diagnosis of lymphoid hyperplasia were $52.9 \%, 81.5 \%$, 27.3\%, and 94.2\%, respectively. Here, Xiao Guang Ni et al. considered type II microvessel pattern as benign and type $\mathrm{V}$ microvessel pattern as malignant.

In summary, NBI endoscopy found to have higher diagnostic values for the diagnosis of NPC in compare to WLE. Specially, NBI is found more sensitive than WLE. Yi Hui Wen et al. found with the higher sensitivity and NPV, narrow-band imaging endoscopy is useful for the early diagnosis of cancerous lesions, improving diagnostic accuracy, and preventing missed diagnosis than WLE. They also concluded that concluded that NBI can find NPC as superficial brown spot which can be missed by WLI. Wang Wen Hung et al. concluded that compared with WLE, NBI can not only provide a timely, convenient and highly reliable assessment of WLE for recurrent nasopharyngeal carcinoma compared with WLE. Haidi Yang et al. found that NBI had a good advantage over WLE in the diagnosis of NPC, with the improvement of sensitivity, specificity as well as positive/negative predictive values. Xiao Guang Ni et al. concluded that NBI endoscopy in compare to WLE could significantly improve the detection of superficial lesions.

\section{Discussion}

Narrowband imaging (NBI, Olympus Medical Systems, Tokyo, Japan) shows potential for early detection of cancer at mucosal sites of head and neck specially in nasopharynx. This technology was developed specifically to highlight the vasculature [7]. The distinction between malignant and non-malignant lesions is the main goal of NPC screening. The NBI filter system is designed for peak absorption spectra of hemoglobin to emphasize capillaries on the mucosal surface. There are many studies proposing NBI classification of microvessels. Many studies found to rely on Xiao Guang Ni et al. [16] NBI classification had good sensitivity, specificity, PPV and NPV for the diagnosis of nasopharyngeal carcinoma. Since NBI significantly improves the contrast of the surface capillary pattern, it is easier to detect small or early malignant lesions, thus improving the diagnostic sensitivity and detection rate and reducing the pathological examination as undifferentiated non-keratinized cancer. Because nasopharynx, which is considered normal during white light endoscopy can hide NPC, NBI endoscopy is likely to assess abnormalities in the nasopharyngeal vasculature, which may indicate subclinical dysplasia or even malignancy [10]. Application of NBI is in many different sites of nasopharynx. These sites have proven their value in detecting early lesions that cannot usually be identified by standard white light endoscopy.

NBI had a promising advantage over conventional WL in the diagnosis of NPC, with the improvement of sensitivity, specificity as well as positive/negative 
predictive values.in comparing to WLE, NBI is found more sensitive in many studies for detecting nasopharyngeal carcinoma which can be used for early diagnosis of cancerous lesions, improve diagnostic accuracy [12]. NBI can find NPC as superficial brown spot which can be missed by WLI. NBI in compare to WLE not only can offer a timely, convenient, and highly reliable assessment of mucosal recurrent nasopharyngeal carcinoma, it can also make endoscopic removal possible.

The NBI system is limited by certain factors, such as visual impairment caused by secretions and darker images overall. During NBI, secretions appear dark green and flicker, which may interfere with screening. In addition, because the image is dark, it may be necessary to place the endoscope very close to the mucosal surface, which may cause contact bleeding and severely interfere with observations [17]. The presence of mucus and blood significantly interfered with the observation of NBI features. The mucus covering the surface of NPC tumor mass is common and in most cases it needs to be cleared to better visualize the nasopharyngeal surface. Contact bleeding caused by fragile cancerous growth poses a major problem in the form of a black coating under NBI light, which obscures the idea of NBI characteristics [16]. To avoid contact bleeding, we can to make good use of this technique. First, it is important to clear the secretions and mucus from the mucosal surface. During observation, the tip of the endoscope should be placed as close to the mucosal surface as possible while avoiding direct contact. Contact bleeding can interfere with observing and masking the microvessel morphology [18]. Second, understanding and mastering the microvascular morphological characteristics of different lesions involve dynamic learning curves also avoid time consumption during procedure with less chance of contact bleeding.

NBI has shown great potential in improving the detection rate of lesions, facilitating better assessment of identified lesions, and reducing the risk of recurrence of lesions requiring resection. There is increasing evidence to recommend its use in colleges and referral clinics that need to evaluate and treat patients with nasopharyngeal mucosal lesions. Nonetheless, further research is needed to realize the full potential of NBI [6]. NBI can be used as a useful visual aid for monitoring potentially malignant lesions due to its video recording capabilities and its ability to highlight small changes in the texture of the mucosal surface and submucosal microvascular system. Longitudinal studies are needed to fully evaluate the effectiveness of NBI in monitoring lesions and whether it can help distinguish between progressive and non-progressive lesions. Further research investigating the types of mucosal and vascular changes in NPC and their correspondence to histopathology is necessary to improve the interpretation of lesions by NBI.

Enhancing the vascular pattern through NBI can simplify the screening of IPCL patterns or changes in the characteristics of mucosal lesions, especially in some atypical flat lesions. Therefore, NBI guided selective biopsy can increase the rate of pathological detection and reduce the chance of oversampling, missed 
diagnosis and misdiagnosis. Selective biopsy can also be used to detect residual or recurrent cancer after surgical intervention or radiotherapy.

\section{Conclusion}

The results of these various studies indicate that the sensitivity, specificity, and accuracy of the NBI model are relatively high in the diagnosis of NPC compared to the WL model. By evaluating the authenticity and reliability of these two modes, it is found that the NBI mode is better than the WL mode. Therefore, we believe that the NBI endoscopy is a worthy diagnostic tool for the diagnosis of NPC.

\section{Conflicts of Interest}

The authors declare no conflicts of interest regarding the publication of this paper.

\section{References}

[1] Lin, Y.C. and Wang, W.H. (2011) Narrow-Band Imaging for Detecting Early Recurrent Nasopharyngeal Carcinoma. Head \& Neck, 33, 591-594. https://doi.org/10.1002/hed.21310

[2] Saba, N.F., et al. (2016) ACR Appropriateness Criteria (R) for Nasopharyngeal Carcinoma. Head and Neck-Journal for the Sciences and Specialties of the Head and Neck, 38, 979-986. https://doi.org/10.1002/hed.24423

[3] Suwondo, S., Surarso, B., and Kristyono, I. (2019) Biopsy Examination Validity Based on Narrow Band Imaging Guidance of Nasopharyngeal Carcinoma Suspected Patients. Indian Journal of Otolaryngology and Head \& Neck Surgery, 71, 395-399. https://doi.org/10.1007/s12070-018-1324-5

[4] Ho, S.H., et al. (2018) Development of Image-Enhanced Endoscopy of the Gastrointestinal Tract: A Review of History and Current Evidences. Journal of Clinical Gastroenterology, 52, 295-306. https://doi.org/10.1097/MCG.0000000000000960

[5] Jang, J.Y. (2015) The Past, Present, and Future of Image-Enhanced Endoscopy. Clinical Endoscopy, 48, 466-475. https://doi.org/10.5946/ce.2015.48.6.466

[6] Filauro, M., et al. (2018) Role of Narrow-Band Imaging in Detection of Head and Neck Unknown Primary Squamous Cell Carcinoma. Laryngoscope, 128, 2060-2066. https://doi.org/10.1002/lary.27098

[7] Garofolo, S., et al. (2015) Intraoperative Narrow Band Imaging Better Delineates Superficial Resection Margins during Transoral Laser Microsurgery for Early Glottic Cancer. Annals of Otology, Rhinology \& Laryngology, 124, 294-298. https://doi.org/10.1177/0003489414556082

[8] Kodashima, S., et al. (2014) Evaluation of a New Image-Enhanced Endoscopic Technology Employing Bandlimited Light for Detection of Esophageal Squamous Cell Carcinoma. Digestive Endoscopy, 26, 164-171.

[9] Robles-Medranda, C., et al. (2019) Detection of Minimal Mucosal Esophageal Lesions in Non-Erosive Gastroesophageal Reflux Disease Using Optical Enhancement plus Optical Magnification. Endoscopy International Open, 7, E979-E986. https://doi.org/10.1055/a-0953-1844

[10] Wang, W.H., et al. (2012) Detection of Mucosal Recurrent Nasopharyngeal Carci- 
nomas after Radiotherapy with Narrow-Band Imaging Endoscopy. International Journal of Radiation Oncology Biology Physics, 83, 1213-1219. https://doi.org/10.1016/j.ijrobp.2011.09.034

[11] Yang, H., et al. (2012) The Diagnostic Value of Narrow-Band Imaging for the Detection of Nasopharyngeal Carcinoma. ORL; Journal for Oto-Rhino-Laryngology and Its Related Specialties, 74, 235-239. https://doi.org/10.1159/000342277

[12] Ho, C.Y., Chan, K.T. and Chu, P.Y. (2013) Comparison of Narrow-Band Imaging and Conventional Nasopharyngoscopy for the Screening of Unaffected Members of Families with Nasopharyngeal Carcinoma. European Archives of Oto-RhinoLaryngology, 270, 2515-2520. https://doi.org/10.1007/s00405-013-2354-y

[13] Larghi, A., Lecca, P.G. and Costamagna, G. (2008) High-Resolution Narrow Band Imaging Endoscopy. Gut, 57, 976-986. https://doi.org/10.1136/gut.2007.127845

[14] Yeung, D.C., et al. (2018) A Meta-Analysis of Narrow-Band Imaging for the Diagnosis of Primary Nasopharyngeal Carcinoma. F1000Research, 7, 759. https://doi.org/10.12688/f1000research.15183.1

[15] Wen, Y.H., et al. (2012) Narrow-Band Imaging: A Novel Screening Tool for Early Nasopharyngeal Carcinoma. Archives of Otolaryngology-Head \& Neck Surgery, 138, 183-188. https://doi.org/10.1001/archoto.2011.1111

[16] Ni, X.G., Zhang, Q.Q. and Wang, G.Q. (2017) Classification of Nasopharyngeal Microvessels Detected by Narrow Band Imaging Endoscopy and Its Role in the Diagnosis of Nasopharyngeal Carcinoma. Acta Oto-Laryngologica, 137, 546-553. https://doi.org/10.1080/00016489.2016.1253869

[17] Ho, C.Y., Lee, Y.L. and Chu, P.Y. (2011) Use of Narrow Band Imaging in Evaluation of Possible Nasopharyngeal Carcinoma. American Journal of Rhinology \& Allergy, 25, 107-111. https://doi.org/10.2500/ajra.2011.25.3582

[18] Madana, J., Lim, C.M. and Loh, K.S. (2015) Narrow Band Imaging of Nasopharynx to Identify Specific Features for Possible Detection of Early Nasopharyngeal Carcinoma. Head \& Neck, 37, 1096-1101. https://doi.org/10.1002/hed.23705

\title{
Abbreviations
}

\author{
WLE-White Light Endoscopy \\ NBI-Narrow Band Imaging \\ IPCLs-Intra Epithelial Papillary Capillary Loops \\ NPC-Nasopharyngeal Carcinoma \\ PPV-Positive Predictive Value \\ NPV-Negative Predictive Value
}

